\title{
REVISÃO ETIOLÓGICA DA LOMBALGIA EM CICLISTAS
}

\author{
ESP. THIAGO AYALA M. DI ALENCAR \\ Graduado em Fisioterapia pela Universidade Estadual de Goiás e especialista em \\ Fisioterapia Traumato-Ortopédica pela UEG \\ Fisioterapeuta da Clínica Fisio Vitale e do Studio Bike Fit (Goiânia - Goiás - Brasil) \\ E-mail: thiagoayala@hotmail.com
}

\section{GRAD. KARINNA F. S. MATIAS}

Graduada em Fisioterapia pela Universidade Estadual de Goiás com formação em

Reeducação Postural Global, Método Philippe Souchard

Fisioterapeuta da Clínica Fisio Vitale e do Studio Bike Fit (Goiânia - Goiás - Brasil)

E-mail: karinnamatias@hotmail.com

\section{MS. RODRIGO R. BINI}

Graduado em Educação Física pela Universidade Federal do Rio Grande do Sul

Mestre em Ciências do Movimento Humano pela Universidade Federal do Rio Grande do Sul Estudante de doutorado na AUT University, Nova Zelândia (North Shore - Auckland - Nova Zelândia)

E-mail: bini.rodrigo@gmail.com

\section{DR. FELIPE PIVETTA CARPES}

Doutor em Ciências do Movimento Humano pela Universidade Federal do Rio Grande do Sul Professor da Universidade Federal do Pampa, Grupo de Pesquisa em Neuromecânica Aplicada

(Uruguaiana - Rio Grande do Sul - Brasil)

E-mail: felipecarpes@unipampa.edu.br

\section{RESUMO}

A dor lombar é uma disfunção comum entre ciclistas e há tempos sua ocorrência tem sido relacionada ao ajuste dos componentes da bicicleta e sua relação com as características anatômicas do ciclista. O objetivo do presente estudo foi revisar os fatores etiológicos da lombalgia em ciclistas considerando artigos das bases de dados SciELO, PubMed e Scopus, publicados de 1965 a 201 I, complementados, quando pertinente, por referências de livros citados por estes artigos. Os resultados indicam que flexão de tronco excessiva, discrepância de comprimento dos membros inferiores, quadro e/ou demais componentes da bicicleta de dimensão inapropriada, falta de ajuste da bicicleta ou ajuste inadequado, fraqueza da musculatura lombo-pélvica, déficit de flexibilidade e desvios posturais pode comprometer o desempenho e o conforto do ciclista sobre a bicicleta, principalmente em percursos de longa distância, podendo levar à lombalgia. Desta forma, o ciclista com lombalgia deve ser avaliado quanto às diferentes variáveis apontadas na literatura de modo a identificar os fatores etiológicos desencadeadores da disfunção lombar para proceder as correções necessárias.

PALAVRAS-CHAVE: Dor; coluna vertebral; ciclismo, longa distância. 


\section{INTRODUÇÃO}

O ciclismo está entre as atividades esportivas com maior número de praticantes no mundo (SALAl et al., 1999) com substancial aumento na última década (ASPLUND; ROSS, 2010). Além de ser utilizada em competições, a bicicleta teve sua inserção entre os meios de locomoção por ser um importante meio de transporte para milhões de pessoas em diversas regiões do mundo (SALAl et al., 1999; SRINIVASAN; BALASUBRAMANIAN, 2007). Mais de 49 milhões de norte americanos utilizam a bicicleta mensalmente, dos quais mais de 5 milhões pedalam pelo menos vinte dias no mês (ASPLUND; ROSS, 20 I 0). No entanto, a prática do ciclismo, ou mesmo o uso da bicicleta como meio de transporte, muitas vezes é prejudicada por lesões por esforço repetitivo, levando à diminuição da frequência de uso da bicicleta (CLARSEN; KROSSHAUG; BAHR, 20 I 0). Dentre estas disfunções, a dor lombar (lombalgia) é uma das mais frequentes, podendo perdurar após o término da prática ciclística, apresentando ocorrência unilateral (SANNER; O'HALLORAN, 2000) ou bilateral (SANNER; O'HALLORAN, 2000; LITTLE; MANSOOR, 2008).

O objetivo desta revisão de literatura foi apontar e discutir os fatores etiológicos da lombalgia em ciclistas, independente do sexo, idade ou tipo de bicicleta utilizada. Este estudo apresenta relevância para treinadores, educadores físicos, fisioterapeutas e médicos que atuam na área esportiva, seja como treinadores ou terapeutas. Embora a lombalgia seja recorrente entre ciclistas, a literatura científica nacional carece de um apanhado sobre este tema. A falta de consenso sobre as possíveis causas da lombalgia em ciclista, assim como já afirmou Pool-Gouzwaard et al. ( 1998) foi um dos motivos que levou à realização desta revisão.

\section{MÉTODOS}

Para seleção dos trabalhos, foram buscados artigos científicos e livros e/ou capítulo sobre ciclismo publicados em datas compreendidas de 1965 a 20 I I . A busca foi realizada nas bases de dados SciELo, PubMed e Scopus utilizando as seguintes palavras chave, por lógica booleana (palavras combinadas por "AND"): dor (pain, douleur, dolor), coluna vertebral (spine, colonne vertébrale, espina), ciclismo (cycling; cyclisme; ciclismo) e longa distância (long distance, longue distance, larga distancia), nos idiomas português, inglês, francês e espanhol, respectivamente. Quando pertinente, utilizou-se referências de livros citados por estes artigos. Dos cinquenta e um documentos utilizados como referência, quarenta e sete eram publicados em 
inglês, um livro era publicado em francês, dois artigos em espanhol e um artigo em português. Dos documentos em inglês, quarenta e três eram artigos científicos, quatro eram capítulos de livros e dois eram livros completos.

\section{RESULTADOS}

A Tabela I apresenta artigos científicos que abordaram fatores etiológicos da lombalgia em ciclistas. Os dados obtidos com os documentos utilizados não possibilitaram apontar qual dos fatores etiológicos é o que mais influência o desenvolvimento da dor lombar. Entende-se por flexão de tronco excessiva e musculatura lombopélvica a postura inadequada do ciclista na bicicleta e o conjunto de músculos que controlam o movimento e estabilizam a coluna lombar e pelve, respectivamente. 
Tabela I. Sumário dos fatores etiológicos encontrados.

\begin{tabular}{|c|c|c|c|c|c|c|c|c|}
\hline \multirow[b]{2}{*}{ Autor } & \multirow[b]{2}{*}{ Ano } & \multicolumn{7}{|c|}{ Fatores etiológicos } \\
\hline & & 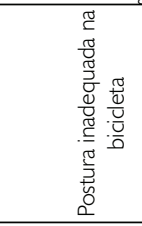 & 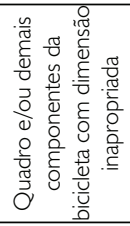 & 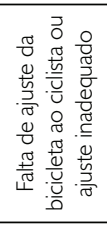 & 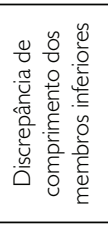 & 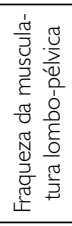 & 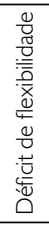 & 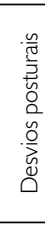 \\
\hline Archer, Hamley e Robson & $1965 *$ & • & & & & & & - \\
\hline McCaw e Bates & $\mid 991$ & & & & - & & & \\
\hline Mellion & 1994 & - & - & - & - & - & - & \\
\hline Manninen e Kallinen & 1996* & $\cdot$ & & & & $\cdot$ & & \\
\hline Usabiaga et al. & 1997* & & & & & • & & \\
\hline Aramendi et al. & 1998* & & & & & - & - & \\
\hline De Vey Mestdagh & 1998 & - & & - & & & & \\
\hline Kronisch & $1998 \mathrm{a}$ & & & $\cdot$ & & & $\cdot$ & \\
\hline Kronisch & $1998 \mathrm{~b}$ & & - & & & & & \\
\hline Salai et al. & 1999* & • & & • & & & & \\
\hline Sanner e O'Halloran & 2000 & - & & - & $\cdot$ & & & \\
\hline Bressel e Larson & $2003 *$ & $\cdot$ & & & & & 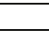 & \\
\hline Burnett et al. & $2004 *$ & & & & & - & & \\
\hline Asplund, Webb e Barkdull & 2005 & & & • & & - & $\bullet$ & \\
\hline Schwellnus e Derman & 2005 & & & $\cdot$ & & - & $\cdot$ & \\
\hline Silberman et al. & 2005 & $\cdot$ & $\cdot$ & & & & & \\
\hline Glasser & 2005 & & & - & & - & • & \\
\hline Pruilt e Matheny & 2006 & & - & • & • & • & $\cdot$ & - \\
\hline Burke e Pruitt & 2006 & & & & $\cdot$ & & & \\
\hline Smurawa & 2006 & • & & & $\cdot$ & & & \\
\hline Martinez & 2006 & & & $\cdot$ & & & & \\
\hline Willardson & 2007 & & & & & - & & \\
\hline Srinivasan e Balasubramanian & $2007 *$ & $\cdot$ & & & & $\cdot$ & & \\
\hline Willy & 2007 & & - & - & & & $\cdot$ & \\
\hline Akuthota et al. & 2008 & & & & & $\bullet$ & & \\
\hline Gómez-Puerto et al. & 2008 & $\cdot$ & $\cdot$ & $\cdot$ & $\cdot$ & & $\cdot$ & \\
\hline Asplund e Ross & 2010 & $\cdot$ & & & & - & & \\
\hline
\end{tabular}


Os artigos científicos apontados na Tabela I indicam que a postura inadequada na bicicleta, a falta de ajuste da bicicleta ao ciclista ou ajuste inadequado e a fraqueza da musculatura lombo-pélvica são os fatores mais frequentemente apontados como causadores de dor lombar em ciclistas.

\section{DISCUSSÃO}

Segundo estudos epidemiológicos, a lombalgia acomete de 30 a $60 \%$ dos ciclistas (WILBER et al., 1995; CALLAGHAN; JARVIS, 1996; CLARSEN; KROSSHAUG; BAHR, 20I0), representando uma das queixas mais comuns entre as disfunções musculoesqueléticas neste esporte (WILBER et al., 1995; CALLAGHAN; JARVIS, 1996; VILLAVICENCIO et al., 2006; LITTLE; MANSOOR, 2008; CLARSEN, KROSSHAUG; BAHR, 20I0). O gênero e a massa corporal são fatores que não apresentam influência sobre a ocorrência de lombalgia em ciclistas, diferente do que ocorre com o fator idade.

Clarsen, Krosshaug e Bahr (2010) realizaram um estudo com cento e nove ciclistas profissionais do campeonato mundial de ciclismo e do Tour de France. Sessenta ciclistas participaram do Tour de France (com idade de $25 \pm 4$ anos) e quarenta e nove do campeonato mundial (com idade de $28 \pm 5$ anos). De acordo com os autores $58 \%(n=63)$ dos ciclistas apresentaram dor lombar durante os últimos doze meses antecedentes à realização da pesquisa, sendo que a incidência foi distribuída da seguinte forma: $27 \%$ com ocorrência de dor fora de temporada, $41 \%$ na pré-temporada e $43 \%$ no início e na alta temporada. Dos 63 ciclistas apenas 45 procuraram assistência médica, sendo que em um ciclista a lombalgia foi intensa o suficiente para obrigá-lo a abandonar o esporte como carreira profissional.

Os resultados encontrados por Clarsen, Krosshaug e Bahr (2010) sugerem que o aumento da incidência da lombalgia está diretamente relacionado ao aumento da intensidade e volume de treinamento. Todavia, como o aumento do volume de treinamento, ou tempo de prática, isoladamente, não levam à lombalgia, acredita-se que os ciclistas apresentam um desequilíbrio muscular que justifique o surgimento do quadro álgico, implicando na ocorrência, até que se prove ao contrário, de pelo menos um dos fatores etiológicos discutidos nesta revisão.

\section{INFLUÊNCIA DA POSTURA SOBRE A LOMBALGIA}

Um dos fatores etiológicos da lombalgia crônica em ciclistas é a flexão de tronco sustentada por longos períodos (MELLION, 1995; CALLAGHAN; JARVIS, 1996; SANNER; O'HALLORAN, 2000; ASPLUND; ST PIERRE, 2004), em especial 
quando o ciclista assume uma postura visando otimização de aspectos aerodinâmicos. O ciclismo da modalidade triathlon é o que apresenta posicionamentos com maior flexão de tronco(MANNINEN; KALLINEN, 1996), seguida pela modalidade estrada (road bike) e montanha (mountain bike). Usabiaga et al. (1997) relatam que o treinamento envolve postura específica à modalidade praticada e adaptação corporal ao esforço físico. Uma postura adequada sobre a bicicleta é fundamental para não incorrer em redução do desempenho ou aumentar o risco de lesão(DE VEY MESTDAGH, 1998; SALAl et al., 1999; SANNER; O'HALLORAN, 2000).

De modo geral, a postura de flexão de tronco utilizada no ciclismo produz uma retificação da lordose lombar, aumenta a tensão do complexo ligamentar posterior e altera a transmissão da pressão sobre os discos intervertebrais, que passam a experimentar aumento da pressão na porção anterior (comprimida) enquanto a posterior é distendida. A distensão da porção posterior do disco e do complexo ligamentar posterior por estimulação mecânica, resultante da postura adotada, induz à lombalgia, pois ambas as estruturas recebem inervação de ramos do nervo sinovertebral e interpretam esta alteração biomecânica como estímulo doloroso. A redução da pressão intradiscal pela redistribuição da sobrecarga imposta ao disco durante a posição de flexão de tronco é alcançada quando o ciclista apoia as mãos sobre o guidão (USABIAGA et al., 1997).

Basicamente três mecanismos podem ser associados à lombalgia em ciclistas (BURNETT et al., 2004). O primeiro, de acordo com Burnett et al. (2004), está relacionado ao fenômeno da flexão-relaxamento, que se manifesta pelo silêncio mioelétrico (não ativação) dos eretores da coluna ao final da amplitude de flexão. Quando as forças musculares são reduzidas, estruturas como ligamentos e discos intervertebrais são colocadas em maior risco de lesão. Enquanto vários ligamentos intervertebrais garantem a estabilização primária das vértebras adjacentes e limitam os movimentos de flexão da coluna vertebral, a musculatura intrínseca e extrínseca à coluna lombar garante a estabilização secundária (ADAMS, 2004). Em segundo lugar, a lombalgia crônica não específica em ciclistas pode resultar em ativação excessiva dos extensores da coluna, resultando em aumento da tensão muscular de toda a coluna lombar. Esse mecanismo foi previamente sugerido como causa da lombalgia crônica não específica (BURNETT et al., 2004). Em terceiro lugar, a flexão prolongada pode ser um importante fator etiológico para a lombalgia, pois a porção posterior do annulus fibrosos pode sofrer microlesões cumulativas (USABIAGA et al., 1997; BURNETT et al., 2004). 


\section{INFLUÊNCIA DO QUADRO E/OU DEMAIS COMPONENTES DA BICICLETA COM A LOMBALGIA}

De acordo com Kronisch ( 1998b) e Trombley (2005), ter uma bicicleta com dimensões que se ajustam bem ao ciclista é importante por proporcionar conforto e ajudar a prevenir lesões musculotendíneas. Uma bicicleta com componentes de dimensões inadequadas ou mal ajustados aumentam a chance de desenvolver lesões por esforço repetitivo em ciclistas (KRONISCH, 1998b).

Para manter uma postura adequada é importante que haja uma harmonia entre as três áreas de contato que o ciclista tem com a bicicleta (Figura I). São elas: as interfaces pelve-selim, mãos-guidão e sapatilha-pedal (COHEN, 1993; DE VEY MESTDAGH, 1998; SILBERMAN et al., 2005; BU et al., 20 I 0; XIANG et al., 20 I I). Embora na figura I não esteja representado, outra possibilidade de posicionamento da mão do ciclista é sobre os manetes e parte superior do guidão. Sem a harmonia entre estas três interfaces o ajuste ideal de uma bicicleta ao ciclista fica comprometido.

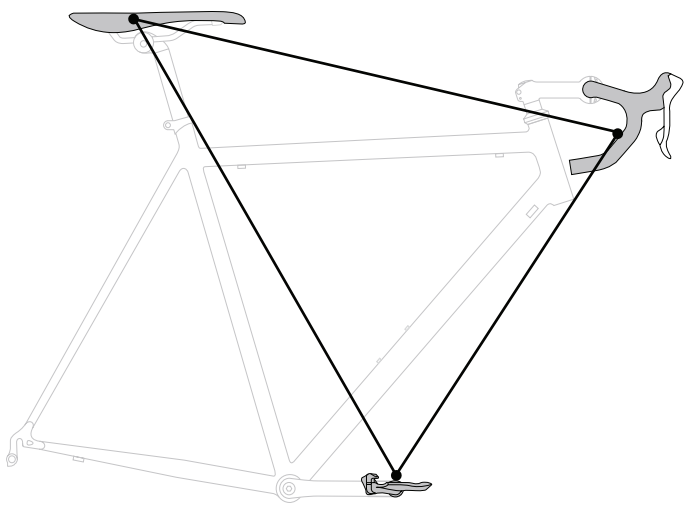

Figura I. Triângulo que representa os três pontos de apoio do ciclista em uma bicicleta para ciclismo de estrada.

Para De Vey Mestdagh ( 1998) a altura do selim, o comprimento do pedivela, o posicionamento do taco na sapatilha e o ajuste ântero-posterior do selim determinam o que pode ser definido como postura relacionada à altura. $\bigcirc$ comprimento e angulação da mesa, a altura, comprimento do guidão e o tubo superior efetivo constituem, juntos, o que se denomina de postura relacionada ao comprimento; em outras palavras, faz referência ao alcance atingido pelo ciclista sobre a bicicleta. 
De acordo com Bressel e Larson (2003) o posicionamento das mãos no guidão, se na patre superior ou sobre o manete, das bicicletas de estrada (Figura I) podem influenciar a amplitude de movimento articular e consequentemente a flexão de tronco.

Músculos da coluna que trabalham em uma posição muito alongada tendem a produzir menor força, comprometendo a manutenção da postura, condição que pode ser responsável por desencadear fadiga muscular e dor. $\bigcirc$ tônus na musculatura paravertebral aumenta ligeiramente durante a prática ciclística realizada sob maior intensidade, todavia, o tônus é dependente, principalmente, da postura do ciclista. Os músculos paravertebrais apresentam menor tônus quando o ciclista mantem-se mais ereto. Em contrapartida, a postura de flexão de tronco diminui a lordose lombar, condição que pode otimizar a contração muscular do psoas, músculo que durante o ciclo da pedalada também tem a função de promover a látero-flexão do corpo vertebral alternadamente entre o lado direito e esquerdo (USABIAGA et al., 1997).

A correção da disfunção apresentada pode envolver o ajuste da bicicleta para que o ciclista pedale em posição de menor flexão de tronco, realizando treinos em trajetos planos para diminuir o esforço muscular dos músculos glúteos e ísquiostibiais (MELLION, 1994; SANNER; O'HALLORAN, 2000). Pode-se minimizar a força realizada pelos músculos ísquios-tibiais, glúteos e da pelve elevando o guidão (SANNER; O'HALLORAN, 2000) ou diminuindo o comprimento da mesa para reduzir a flexão de tronco (MELLION, 1994; SANNER; O'HALLORAN, 2000; ASPLUND; WEBB; BARKDULL, 2005).

\section{INFLUÊNCIA DO AJUSTE DA BICICLETA PARA PREVENIR A OCORRÊNCIA DE LOMBALGIA}

Ojuste da bicicleta às características corporais do ciclista, procedimento conhecido nacional e internacionalmente por bike fit, envolve avaliação postural e biomecânica do ciclista sobre a bicicleta, sendo procurado pelos ciclistas para prevenir lesões, aumentar o conforto e o desempenho (DI ALENCAR; MATIAS, 2009). A prevenção da lombalgia está relacionada ao ajuste adequado da bicicleta ao ciclista (MELLION, I994; ASPLUND; ST PIERRE, 2004; SCHWELLNUS; DERMAN, 2005; ASPLUND; WEBB; BARKDULL, 2005; PRUITT; MATHENY, 2006). Todavia, se um ciclista já sofre de lombalgia, um ajuste da bicicleta realizado de forma inadequado pode agravar o quadro de dor (PRUITT; MATHENY, 2006).

ajuste pode não ser suficiente para resolver problemas relacionados à lombalgia quando o ciclista possui uma bicicleta com quadro e/ou mesa e/ou tubo 
superior efetivo (effective top tube) (Figura 2), de dimensão inapropriada às características antropométricas do ciclista (MELLION, 1994; KRONISCH, 1998a), ou ainda a um guidão muito baixo(MELLION, 1994; KRONISCH, I 998a; ASPLUND; ST PIERRE, 2004; GLASSER, 2005), condição que não permite ajuste na altura devido à espiga do garfo ou suspensão ser demasiada curta (PRUITT; MATHENY, 2006). Um quadro de tamanho inapropriado ao ciclista pode dificultar, ou até mesmo comprometer, o ajuste por não permitir a realização dos ajustes necessários. Antropometria, dimensão, ajuste e postura sobre a bicicleta são variáveis que se correlacionam (SCHULTZ; GORDON, 20l0; BU et al., 20l0), e até a elaboração deste artigo não consta nenhuma pesquisa que tenha associado as variáveis entre si e com a ocorrência de lombalgia em ciclistas. Schultz e Gordon (20 l 0) defendem a realização de um estudo que associe a antropometria do ciclista às dimensões da bicicleta e ajuste bem como a postura sobre a mesma, pois são variáveis que se correlacionam.

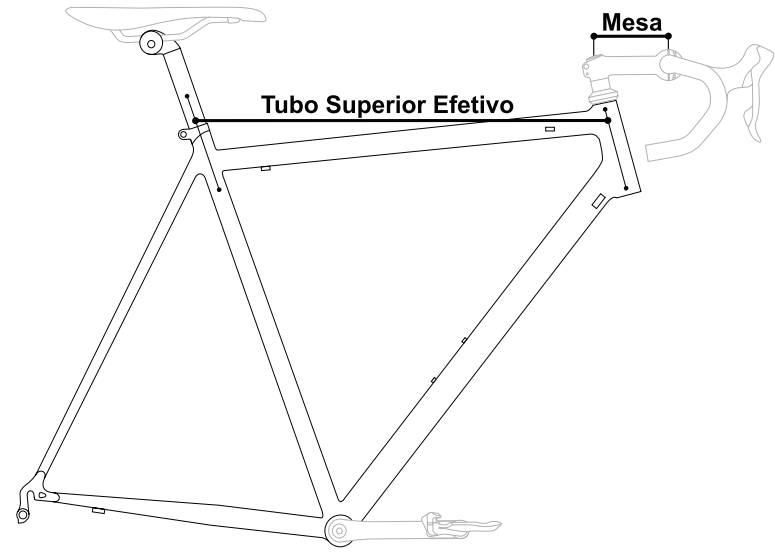

Figura 2. Representação do tubo superior efetivo e da mesa em um quadro de bicicleta para ciclismo de estrada.

Salai et al. ( 1999) relatam que a incidência e gravidade de lombalgia pode ser reduzida em $72 \%$ dos casos se a extremidade anterior do selim for inclinada de 10 a $15^{\circ}$ para baixo. A alteração da inclinação do selim como conduta para resolver a lombalgia é questionada por Goméz-Puerto et al. (2008) e pode, ainda, desencadear dor nas mãos (KRONISCH, I998a; SILBERMAN et al., 2005) por aumentar a pressão na região tenar e hipotenar. Por outro lado, um guidão relativamente baixo pode estar relacionado a um reduzido ângulo da mesa (PRUITT; MATHENY, 2006) e ainda ser o responsável por acentuar a flexão de tronco, aumentando a tensão nas 
estruturas musculoesqueléticas da região lombar (ASPLUND; WEBB; BARKDULL, 2005). Garantir a adequada altura do guidão, do comprimento do tubo superior efetivo e flexão de tronco do ciclista ajuda a minimizar ou até mesmo eliminar a lombalgia (MELLION, I994; ASPLUND; WEBB; BARKDULL, 2005).

De acordo com Mellion (1994) e Bouché, Vincent e Sullivan (2005), é importante conferir se a bicicleta encontra-se devidamente ajustada, pois esta prática complementa a avaliação clínica do ciclista. Estabelecer um diagnóstico específico e identificar fatores etiológicos são condutas que determinam a escolha do tratamento mais adequado.

\section{INFLUÊNCIA DA ESTABILIZAÇÃO CENTRAL E MOBILIDADE ARTICULAR SOBRE A LOMBALGIA}

Embora ciclistas possam ser vulneráveis à ocorrência de lombalgia, existem poucas evidências de anormalidades radiográficas na maioria das disfunções lombares dolorosas, implicando em um diagnóstico de lombalgia crônica não específica (BURNETT et al., 2004). A sacroileíte, embora cause lombalgia, deve ser avaliada separadamente para um diagnóstico mais apropriado (SMURAWA, 2006).

A fraqueza da musculatura lombo-pélvica pode levar à fadiga precoce e lombalgia(MANNINEN; KALLINEN, 1996; SRINIVASAN; BALASUBRAMANIAN, 2007; WILLARDSON, 2007; AKUTHOTA et al., 2008) em ciclistas que realizam provas de longa distância como o Race Across America, quando o estresse imposto à musculatura estabilizadora da coluna lombar supera a capacidade de produção de força (GRIFFITHS-FABLE, 2006; ASPLUND; ROSS, 20 I0).

A região lombo-pélvica é uma zona de transição de forças entre os membros superiores e inferiores. $\bigcirc$ ciclista com boa estabilidade lombo-pélvica consegue transferir as forças produzidas pelos membros inferiores ao pedal com maior eficiência (USABIAGA et al., 1997; WILLARDSON, 2007; ASPLUND; ROSS, 20 I0), sem gerar perda de energia por desequilíbrio musculoesquelético e, consequentemente, sem sobrecarregar a coluna lombar (WILLARDSON, 2007). Além disso, o ciclista com bom condicionamento da musculatura lombo-pélvica pode suportar a postura aerodinâmica por mais tempo sem resultar em sensação de desconforto (ASPLUND; ROSS, 2010).

Asplund e Ross (2010) estabeleceram uma relação entre condicionamento da musculatura lombo-pélvica com a elevação do guidão, a partir de evidências baseadas na prática. Para os autores, o desempenho na manutenção na posição de plank (prancha) é um parâmetro para ponderar o ajuste da altura do guidão. 
O exercício de plank, citado por Asplund e Ross (20 I0), se resume em apoiar as pontas dos pés e o antebraço ao chão, mantendo o cotovelo e ombro fletidos a $90^{\circ}$. A Tabela 2 correlaciona o tempo mantido pelo ciclista na posição de plank com o condicionamento lombo-pélvico (resultado) e a tolerância aos ajustes na altura do guidão.

Tabela 2. Relação entre o tempo de permanência na posição de plank e tolerância ao ajuste de altura do guidão

\begin{tabular}{lll}
\hline Tempo & Resultado & Tolerância aos ajustes significativos de altura do guidão \\
\hline$<$ I minuto & Razoável & Baixa \\
de I a 2 minutos & Bom & Moderada \\
$>2$ minutos & Excelente & Alta \\
\hline
\end{tabular}

Srinivasan e Balasubramanian (2007) realizaram um estudo com 14 ciclistas do sexo masculino (idade média de $25 \pm 2$ anos e massa corporal de $64 \pm 8,9 \mathrm{~kg}$ ) usuários de bicicleta de estrada. Os participantes foram divididos em dois grupos pareados, sendo que um dos grupos foi formado por ciclistas que apresentavam histórico de lombalgia sem relação com lesão prévia. Os músculos avaliados por meio da eletromiografia de superfície foram o bíceps braquial, trapézio (fibras mediais), grande dorsal (fibras mediais) e eretor da espinha, todos bilateralmente. A ativação muscular foi mensurada antes de pedalar, e após 15 e 30 minutos de pedalada. Os resultados revelaram diferenças significativas para a ativação muscular do trapézio e eretores da espinha direito do grupo com lombalgia comparado com o grupo sem lombalgia (controle). A interpretação dos resultados sugere maior fadiga dos músculos posteriores do tronco no grupo com lombalgia. A possibilidade de que o aumento na fadiga muscular possa agravar a dor lombar deve ser considerada a partir dos resultados deste estudo, de forma que a importância do fortalecimento da musculatura lombo-pélvica passe a ser salientada (ASPLUND; WEBB; BARKDUL, 2005; AKUTHOTA et al., 2008).

Hides, Richardson e Jull (1996) relatam casos dos músculos multífidos com hipotrofia em pessoas com lombalgia, músculo que para Burnett et al. (2004) apresenta função chave na estabilização da coluna lombar. De acordo com os autores, esta hipotrofia muscular pode ser resultado da contração muscular reflexa dos músculos paravertebrais induzida pela estimulação mecânica do complexo ligamentar posterior e da porção posterior do disco intervertebral quando o ciclista se encontra com tronco fletido. Como a postura de flexão de tronco é mantida por longos períodos durante a prática ciclística, a tendência é que os músculos que 
realizam a estabilização e extensão da coluna vertebral entrem em fadiga, diminuindo a resistência do atleta para a manutenção da postura (USABIAGA et al., 1997). Desta forma, o fortalecimento da musculatura lombo-pélvica e o alongamento de músculos dos membros inferiores ajuda a prevenir a ocorrência de disfunções lombares e aumenta o desempenho na prática esportiva(MELLION, 1994; ASPLUND; WEBB; BARKDULL, 2005).

Se a musculatura da cadeia posterior do tronco não está bem condicionada e inapta a manter a flexão de tronco (KRONISCH, 1998a; SCHWELLNUS; DERMAN, 2005; ASPLUND; WEBB; BARKDULL, 2005), a fadiga muscular e a tensão excessiva gerada podem resultar em dor(ASPLUND; WEBB; BARKDULL, 2005). Paralelamente, pedalar com marcha pesada ou em aclives por longo período pode fadigar precocemente o glúteo máximo e ísquios-tibiais (ASPLUND; WEBB; BARKDULL, 2005) promovendo uma anteroversão pélvica, aumentando a tensão sobre a musculatura lombar e resultando em dor lombar (ASPLUND; WEBB; BARKDULL, 2005). O posicionamento ântero-posterior da pelve é controlado pelo equilíbrio entre a musculatura lombo-pélvica (Figura 3) (MELLION, I994).

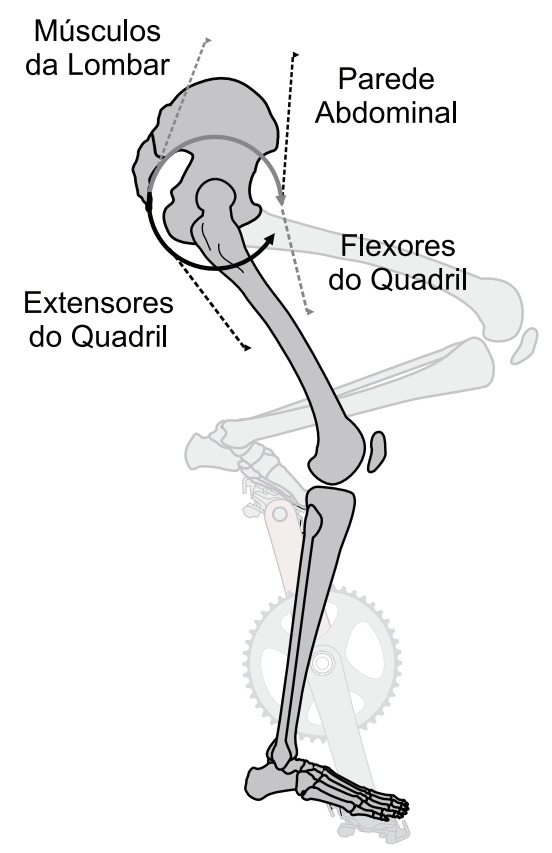

Figura 3. Equilíbrio lombo-pélvico durante a pedalada. A semicircunferência em cinza representa a anteroversão e a preta a retroversão pélvica. 
O quadríceps, especialmente o reto femoral e o liliopsoas atuando em elevada tensão, tende a realizar uma anteroversão da pelve (MELLION 1994; ASPLUND; WEBB; BARKDULL, 2005). Por outro lado, se os ísquios-tibiais estão tensos, a anteroversão pélvica é restringida, resultando em aumento da flexão da coluna lombar (MELLION, 1994; ASPLUND; WEBB; BARKDULL, 2005). Embora a flexão do tronco aumentada resulte em uma vantagem mecânica para os glúteos hiperestenderem a coluna lombar, esta postura associada à grande extensão do joelho no ciclo da pedalada eleva a tensão dos glúteos, que, consequentemente, tentam hiperestender a coluna lombar resultando em dor (SANNER; O'HALLORAN, 2000). Little e Mansoor (20 I 0) relatam um caso de síndrome do estalido no quadril de um ciclista profissional de quarenta e oito anos. Esta disfunção foi descrita pelo ciclista durante avaliação, como um desconforto à flexo-extensão do quadril associado à lombalgia. De acordo com os autores tratava-se de um ressalto do tendão do músculo íliopsoas sobre a região óssea chamada eminência iliopectínea, na passagem da extensão para a flexão do joelho. Este ressalto é responsável por elevar à tensão do íliopsoas e aumentar o estresse na coluna lombar.

\section{INFLUÊNCIA DA DISCREPÂNCIA DE COMPRIMENTO DOS MEMBROS INFERIORES SOBRE A LOMBALGIA}

A discrepância de comprimento dos membros inferiores, estrutural ou funcional (MCCAW; BATES, I99I; BURKE; PRUITT, 2003; PRUITT; MATHENY, 2006), é outro fator etiológico da lombalgia em ciclistas (MELLION, 1994; SANNER; O'HALLORAN, 2000; PRUITT; MATHENY, 2006; BURKE; PRUITT, 2006; GÓMEZ-PUERTO et al., 2008). A discrepância estrutural deve-se à ocorrência de membros inferiores com diferentes comprimentos, enquanto a discrepância de origem funcional resulta de uma adaptação funcional, como pronação do arco plantar longitudinal (BOLZ; DAVIES, 1984; MCCAW; BATES, 199।; PRUITT; MATHENY, 2006), ou mesmo desequilíbrio na produção de força aplicada no pedal (CARPES et al. 20 I 0). A discrepância estrutural é corrigida utilizando um calço entre o taco e o solado da sapatilha (MELLION, 1994; PRUITT; MATHENY, 2006).

O uso de calço para correção da discrepância de comprimento ultrapassou a indicação para a população em geral e passou a ser realidade nos esportes, como corrida e ciclismo. Pruitt (2006) relata um caso de discrepância de comprimento de membros inferiores em Eddy Merckx, condição que pode ter sido a causa do desconforto que o ciclista sentia ao pedalar e que poderia ter sido corrigida pelo uso de calço. Merckx foi um ciclista profissional que ganhou destaque no esporte pelo seu grande desempenho e pelo hábito de ajustes sucessivos na altura do selim durante as provas. 
Para avaliar a influência dos calços na correção da discrepância no comprimento dos membros inferiores e subsequente alívio de dor lombar em não-ciclistas Golightly et al. (2007) realizaram um estudo com doze voluntários com histórico de lombalgia e discrepância de comprimento variando de um a trinta anos e 6,4 a $22,2 \mathrm{~mm}$, respectivamente. Os autores observaram que a correção de $61 \pm 24 \%$ da discrepância pelo uso de calço resultou em diminuição da dor lombar em 75\% dos participantes. De acordo com Brady et al. (2003), o alívio da dor lombar em pessoas de mais idade com presença de discrepância tende a ser menos pronunciável quando comparado a pessoas jovens, provavelmente porque apresentam compensação já estruturada. Para a melhor adaptação e controle do alívio dos sintomas na população em geral e em corredores, Brady et al. (2003) e Golightly et al. (2007) recomendam a indicação de calço para correção gradual, colocadas no interior do calçado, podendo ainda ser providenciada no solado também.

Uma realidade semelhante a dos corredores ocorre entre ciclistas. Como o ciclista com discrepância de comprimento dos membros inferiores apresenta uma inclinação pélvica em relação ao membro mais curto, esta diferença pode levar à formação de uma escoliose lombar compensatória de convexidade direcionada ao lado do membro mais curto e compressão do disco intervertebral no lado da concavidade. Esta característica biomecânica alterada favorece a protusão discal e pinçamento da raiz nervosa (MCCAW; BATES, 199I). De acordo com McCaw e Bates ( 199 |), quanto maior a discrepância de comprimento dos membros inferiores maior é o grau de escoliose. Além disso, a convexidade lombar produz um padrão de assimetria da atividade muscular em paravertebrais, tensor da fáscia lata e ísquios-tibiais, sendo a maior atividade ipsilateral ao membro mais comprido, e consequentemente, à concavidade do desvio gerado (BOLZ; DAVIES, 1984).

A discrepância de comprimento dos membros inferiores é responsável também por aumentar o risco de osteoartrite do quadril, ciatalgia, bursite trocanteriana e síndrome da banda ílio-tibial no membro de maior comprimento, resultando em tensionamento excessivo da fáscia lata, promovido pela inclinação pélvica sobre o trocânter maior e côndilo femoral lateral (BOLZ; DAVIES, I 984; MCCAW; BATES, 199I). A bursite trocanteriana (SANNER; O'HALLORAN, 2000) e a síndrome da banda ílio-tibial(SANNER; O'HALLORAN, 2000) também podem decorrer de um encurtamento do glúteo máximo, visto que este músculo apresenta inserção na banda ílio-tibial. Neste caso, ciclistas que aplicam tensão excessiva sobre a fáscia lata, podem aumentar a fricção sobre o trocânter maior e côndilo femoral lateral. 
A indicação de correção da discrepância de comprimento dos membros inferiores é determinada a partir da magnitude da assimetria diagnosticada pela mensuração da distância da espinha ilíaca ântero-superior ao maléolo medial (BRADY et al., 2003; DI ALENCAR; MATIAS, 2009) ou por meio de um exame de imagem denominado por escanometria (BRADY et al., 2003; BURKE; PRUITT, 2003; PRUITT; MATHENY, 2006). Enquanto para Callaghan (2005) a discrepância maior ou igual a 6,4 mm é caracterizada como significativa, Burke e Pruitt (2003) relatam que a correção de discrepância menor ou igual a 4 mm só necessita de correção em casos sintomáticos.

Em posição ortostática a pessoa com discrepância de comprimento compensa o desequilíbrio dos membros flexionando ligeiramente o joelho do membro mais comprido (BOLZ; DAVIES, 1984). Este mecanismo de compensação justifica a conduta defendida por Burke e Pruitt (2003) para corrigir a discrepância de comprimento em ciclistas. Burke e Pruitt (2003) relatam que se o ciclista apresenta uma discrepância de $6 \mathrm{~mm}$, a correção é feita colocando um calço de $3 \mathrm{~mm}$ entre o taco e a sapatilha, associado à posteriorização do taco em I mm na sapatilha do membro mais comprido (levando o pé para frente) e anteriorização em I mm na sapatilha do membro mais curto (levando o pé para trás). Para estes autores, um ciclista com discrepância inferior a $6 \mathrm{~mm}$ deve ter o taco movido para frente de I a $2 \mathrm{~mm}$ no membro mais curto ou para trás de I a $2 \mathrm{~mm}$ no membro mais comprido.

Em situações nas quais a discrepância ultrapasse 6 mm, a correção é feita por meio da utilização de calço de material resistente à deformação por compressão, instalado entre o taco e o solado da sapatilha (PRUITT; MATHENY, 2006), por ser esta a zona de contato do pé com o pedal. Recomenda-se a utilização de calços para correção de até $50 \%$ da discrepância diagnosticada, pois em muitos casos a estrutura musculoesquelética já buscou se adaptar por meio de compensação biomecânica (e.g. alteração dos ângulos articulares) e a realização de uma correção total poderia trazer mais desconforto ao invés de alívio (BRADY et al., 2003; PRUITT; MATHENY, 2006; GOLIGHTLY et al., 2007).

Bolz e Davies ( 1984) relatam que em pessoas com discrepância de membros inferiores o membro inferior mais curto apresenta menor capacidade de produzir força. Partindo deste achado é possível hipotetizar que além da alteração biomecânica proveniente da discrepância espera-se que o membro mais comprido aplique mais força ao pedal, resultando em assimetria de torque, sobrecarregando a musculatura do membro inferior e levar à tensão lombo-pélvica ipsilateral ao membro mais comprido. Há carência de estudos até o momento proporcionando evidências para estas hipóteses. 
Define-se por flexibilidade a habilidade que uma articulação tem de alcançar toda a amplitude de movimento pré-estabelecida anatomicamente. As estruturas que podem limitar a amplitude de movimento são as estruturas ósseas adjacentes a articulação, os ligamentos, a cápsula articular e os músculos. A causa mais comum de déficit de flexibilidade é o encurtamento muscular, alteração musculotendínea que pode ser revertida com a realização de alongamento muscular (FEINGOLD, 1986). Feingold (1986) relata que o déficit de flexibilidade está associado a um aumento das lesões relacionadas à tensão muscular, pois uma diminuição da amplitude de movimento em uma articulação exige compensação em outra. Em outras palavras, se uma articulação não apresenta amplitude de movimento satisfatória para a postura a ser adotada, outros segmentos corporais serão forçados a aumentar a amplitude de movimento como um mecanismo compensatório, aumentando consequentemente a tensão da estrutura musculoesquelética envolvida (FEINGOLD, 1986).

O ciclismo é um esporte de movimentos repetitivos e sustentados por longos períodos. Esta característica do esporte associada à amplitude de movimento articular que não permite aos músculos biarticulares retomar o comprimento muscular típico da posição anatômica (GRAU, 2008) somada à negligência da prática de alongamento por parte dos ciclistas, favorece o encurtamento de músculos biarticulares (SANNER; O'HALLORAN, 2000). O encurtamento muscular contribui ainda com a alteração postural, como por exemplo, anteroversão ou retroversão pélvica (GLASSER, 2005). Estudos são necessários para indicar a frequência de realização de exercícios de alongamento muscular para ciclistas, com e sem histórico de lesões com fins de redução do quadro álgico.

\section{INFLUÊNCIA DE DESVIOS POSTURAIS SOBRE A LOMBALGIA}

Adicionalmente aos fatores etiológicos já discutidos, as alterações posturais como hipercifose (ARCHER; HAMLEY; ROBSON, 1965; MESTDAGH, 1998), hiperlordose (ARCHER; HAMLEY; ROBSON, I 965; PRUITT; MATHENY, 2006; GÓMEZ-PUERTO et al., 2008) e escoliose(ARCHER; HAMLEY; ROBSON, I965; MCCAW; BATES, 199I; PRUITT; MATHENY, 2006; GÓMEZ-PUERTO et al., 2008) também são fatores em potencial para o desenvolvimento de dor lombar em ciclistas. A causa da dor lombar decorrente destes desvios posturais pode estar relacionada à compressão do disco intervertebral (MANNINEN; KALLINEN, 1996; SCHWELLNUS; DERMAN, 2005; PRUITT; MATHENY, 2006), tração nas cápsulas 
articulares, resultando em tensão muscular ou deformação ligamentar(USABIAGA et al., 1997; SCHWELLNUS; DERMAN, 2005). A correção dos desvios posturais pode ser conduzida por meio do método de Reeducação Postural Global, o qual prioriza o equilíbrio das cadeias musculares (GRAU, 2008).

Determinar a altura adequada do selim permite que a coluna lombar e a articulação do quadril sejam utilizados em suas amplitudes de movimento ideais (MELLION, 1994; GRIFFITHS-FABLE, 2006), possibilitando atingir maior eficiência dos músculos do tronco, sem, contudo, levá-los à tensão excessiva ou fadiga (GRIFFITHS-FABLE, 2006). Além disso, o selim elevado em demasia, para o ciclista com escoliose tóraco-lombar, por exemplo, aumenta o desconforto, dado o movimento de báscula látero-lateral do quadril para manter o contato com o pedal durante o ciclo da pedalada (ASPLUND; ST PIERRE, 2004). O movimento de báscula também pode decorrer da presença de discrepância de comprimento de membros inferiores (ASPLUND; ST PIERRE, 2004).

Dado a possibilidade de ocorrência de discrepância no comprimento dos membros inferiores, fraqueza da musculatura lombo-pélvica, déficit de flexibilidade e desvios posturais em ciclistas como fator etiológico da lombalgia, faz-se necessário a realização da avaliação antropométrica e musculoesquelética anterior à realização do ajuste da bicicleta ou elaboração da conduta de tratamento (DI ALENCAR; MATIAS, 2009).

\section{CONCLUSÃO}

A lombalgia é uma disfunção musculoesquelética que tem comprometido o desempenho de ciclistas ao longo dos anos, tanto em treinamentos quanto em competições. Os fatores etiológicos da lombalgia apontados na literatura foram a flexão de tronco excessiva, o quadro e/ou demais componentes da bicicleta com dimensão inapropriada, a falta de ajuste da bicicleta ao ciclista ou o ajuste inadequado, a discrepância de comprimento dos membros inferiores, a fraqueza da musculatura do lombo-pélvica, o déficit de flexibilidade e desvios posturais. A realização do ajuste da bicicleta de forma adequada é importante para minimizar ou até mesmo excluir o efeito do posicionamento inadequado na bicicleta sobre a ocorrência de lesões e dor lombar, disfunção que apresenta alta incidência em ciclistas, que pode comprometer o desempenho e levar ao abandono do esporte. As diferenças experimentais dos estudos avaliados e a robustez das evidências apresentadas foram fatores que limitaram para recomendações mais específicas quanto aos métodos de prevenção da lombalgia em ciclistas. 


\section{Etiologic review of low back pain in cyclists}

ABSTRACT: Low back pain is a common disorder in cyclists and it's occurrence has long been related mainly to a lack of flexibility. The aim of this study was to review the etiologic factors of low back pain in cyclists cited by articles from SciELO, PubMed and Scopus, published from 1965 to 2011 , indicated, when appropriate, by books cited in these articles. The results indicated that excessive trunk flexion, leg length discrepancy, frame size and/or other components of inappropriate length, poor bike fit or improper bike fit, lumbopelvic muscle weakness, poor flexibility and postural deviations may compromise performance and comfort of the cyclist on the bike, especially during long distance riding and may lead to the development of low back pain. Therefore, the cyclist with back pain should be evaluated based on different variables described in literature with the purpose of identifying the etiologic factors may trigger lumbar dysfunction to make the necessary corrections.

KEYWORDS: Etiology; low back pain; cycling; long distance.

\section{Revisión etiológica de la lumbalgia en ciclistas}

RESUMEN: El dolor lumbar es un trastorno frecuente en los ciclistas y su presencia hace mucho tiempo es referida principalmente a la falta de flexibilidad. El objetivo de este estudio fue analizar los factores etiológicos de dolor lumbar en ciclistas con base en artículos de SciELO, PubMed y Scopus, publicados desde 1965 a 201 I, complementados, cuando necesario, por las referencias citadas en estos artículos. Los resultados indican que la flexión demasiada del tronco, discrepancia en la longitud de las piernas, la dimensión inadecuada del cuadro y los otros componentes de la bicicleta, la falta de ajuste de la bicicleta o ajuste incorrecto, debilidad muscular en el cuadril, déficit de la flexibilidad y desviaciones posturales pueden comprometer el rendimiento y la comodidad del ciclista sobre la bicicleta, especialmente en largas distancias y puede conducir al desarrollo de la dolor lumbar. Así, el corredor con el dolor lumbar debe ser evaluado en respecto a las diferentes variables descritas en la literatura con el fin de identificar los factores etiológicos que provocan la disfunción lumbar para hacer las correcciones necesarias.

PALABRAS CLAVE: Etiología; dolor lumbar; ciclismo; larga distancia.

\section{REFERÊNCIAS}

ADAMS, M. A. Biomechanics of back pain. Acupuncture in Medicine, v. 22, n. 4, p. 178-188, 2004.

AKUTHOTA, V. et al. Core stability exercise principles. Current Sports Medicine Reports, v. 7, n. I, p. 39-44, 2008.

ARAMENDI, J. F. et al. La columna vertebral lumbar en las diferentes posiciones del ciclismo profesional en ruta. Archivos de medicina del deporte, v. XV, n. 66, p. 343-347, 1998. 
ARCHER, J.; HAMLEY, E. J.; ROBSON, H. E. The fitness assessment of competitive cyclists. Britsh Association of Sport and Medicine, v. 2, n. 3-4, p. 90-100, 1965.

ASPLUND, C.; ROSS, M. Core stability and bicycling. Current Sports Medicine Reports, v. 9, n. 3, p. $155-160,2010$

ASPLUND, C.; ST PIERRE, P. Knee pain and bicycling - fitting concepts for clinicians. The Physician and Sportsmedicine, v 32, n 4, p. 23-30, 2004.

ASPLUND, C.; WEBB, C.; BARKDULL, T. Neck and Back Pain in Bicycling. Current Sports Medicine Reports, v 4, n 5, p. 27I-274, 2005.

BOUCHÉ, R. T.; VINCENT, P. M.; SULLIVAN, K. Bike fit evaluation: can it help diagnose and prevent cycling injuries? Podiatry Today, v. 19, n. 12, p. 28-34, 2006.

BOLZ, S.; DAVIES, G. J. Leg length differences and correlation with total leg strength. The Journal of Orthopaedic and Sports Physical Therapy, v. 6, n. 2, p. 123-129, 1984.

BRADY, R. J. et al. Limb length inequality: clinical implications for assessment and intervention. Journal of Orthopaedic \& Sports Physical Therapy, v. 33, n. 5, p. $221-234,2003$.

BRESSEL, E., LARSON, B. J. Bicycle seat designs and their effect on pelvic angle, trunk angle, and comfort. Medicine \& Science in Sports \& Exercise, v. 35, n. 2, p. 327-332, 2003.

BU, Y. et al. Optimal Design of Mountain Bicycle Based on Biomechanics. Transactions of Tianjin University, v. 16, n. I, p. 45-49, 2010.

BURKE, E. R.; PRUITT, A. L. "Body Positioning for Cycling". In: Burke ER. High-Tech Cycling. 2nd ed. Champaign, IL: Human Kinetics, 2003, p. 69-92.

BURNETT, A. F. et al. Spinal kinematics and trunk muscle activity in cyclists: a comparison between healthy controls and non-specific chronic low back pain subjects - a pilot investigation. Manual Therapy, v. 9, n. 4, p. 211-219, 2004.

CALLAGHAN, M. J. Lower body problems and injury in cycling. Journal of Bodywork and Movement Therapies, v. 9, n. 3, p. 226-236, 2005.

CALLAGHAN, M. J.; JARVIS, C. Evaluation of elite british cyclists: the role of the squad medical. British Journal in Sports Medicine, v. 30, n. 4, 349-353, 1996.

CARPES, F. P.; MOTA, C. B.; et al. On the bilateral asymmetry during running and cycling - A review considering leg preference. Physical Therapy in Sport, v. II, n. 4, p. 136-142, 2010.

CLARSEN, B.; KROSSHAUG, T.; BAHR, R. Overuse injuries in professional road cyclists. The Americam Journal of Sports Medicine, v. 38, n. 12, p. 2494-250 I, 2010.

COHEN, G. C. Cycling injuries. Canadian Family Physician, v. 39, p. 628-632, 1993. 
DE VEY MESTDAGH, K. Personal perspective: in search of an optimum cycling posture. Applied Ergonomics, v. 29, n. 5, p. 325-334, 1998.

DI ALENCAR, T. A. M.; MATIAS, K. F. S. Importância da avaliação musculoesquelética e biomecânica para o bike fit. Revista Movimenta, Goiânia, v. 2, n. 3, p. 84-92, 2009.

DI ALENCAR, T. A. M.; MATIAS, K. F. S. Princípios fisiológicos do aquecimento e alongamento muscular na atividade esportiva. Revista Brasileira de Medicina do Esporte, São Paulo, v. 16, n. 3, p. 230-234, mai/jun. 2010.

FEINGOLD, M. L. "Flexibility standards of the U. S. cycling team". In: BURKE, E. R (org.). Science of Cycling. Champaign: Human Kinetics, 1986. p. 47-68.

GLASSER, L. L. The spine and bicycling: new perspectives. In: LENNARD, T. A.; CRABTREE, H. M (org.). Spine in sports. Philadelphia: Elsevier, 2005. p. II3-133.

GOLIGHTLY, Y. M. et al. Changes in pain and disability secondary to shoe lift intervention in subjects with limb length inequality and chronic low back pain: a preliminary report. Journal of Orthopaedic \& Sports Physical Therapy, v. 37, n. 7, pp. 380-388, 2007.

GÓMEZ-PUERTO, J. R. et al. La importancia de los ajustes de la bicicleta en la prevención de las lesiones en el ciclismo: aplicaciones prácticas. Revista Andaluza de Medicina del Deporte, v. I, n. 2, p. 73-8I, 2008.

GRAU, N. Le stretching global actif au service du geste sportif. Impressions Nouvelles, 2008. GRIFFITHS-FABLE, S. Injury prevention: indoor cycling. IDEA Fitness Journal, v. 3, n. 8, p. $1-2,2006$.

HIDES, J. A.; RICHARDSON, C. A.; JULL, G. A. Multifidus muscle recovery is not automatic after resolution of acute, first-episode low back pain. Spine, v. 2 I, n. 23, p. 2763-2769, 1996.

KRONISCH, R. L. Mountain biking injuries: fitting treatment to the causes. The Physician Sportsmed, v. 26, n. 3, p. 64-70, 1998 a.

KRONISCH, RL. How to fit a mountain bike. The Physician and Sportsmedicine, v. 26, n. 3, p. I-4, 1998b.

LITTLE, T. L.; MANSOOR, J. Low Back Pain Associated with Internal Snapping Hip Syndrome in a Competitive Cyclist. British Journal of Sports Medicine, 2008; 42(4): 308-9.

MCCAW, S. T.; BATES, B. T. Biomechanical implications of mild leg length inequality. British Journal of Sports Medicine, v. 25, n. I, p. 10-13, 1991.

MANNINEN, J. S.; KALLINEN, M. Low back pain and other overuse injuries in a group of japanese triathletes. British Journal of Sports Medicine, v. 30, n. 2, p. I34- I39, 1996. 
MARTINEZ, J. M. Medical coverage of cycling events. Current Sports Medicine Reports, v. 5, n. 3, p. 125-130, 2006.

MELLION, M. B. Neck and back pain in bicycling. Clinical Sports Medicine, v. I3, n. I, p. |37-164, 1994.

POOL-GOUZWAARD, A. L. et al. Insufficient lumbopelvic stability: a clinical, anatomical and biomechanical approach to 'a-specific' low-back pain. Manual Therapy, v. 3, n. I, p. 12-20, 1998.

PRUITT, A. L.; MATHENY, F. Andy Pruitt's complete medical guide for cyclists. Boulder, CO: VeloPress, 2006.

SALAI, M. et al. Effect of changing the saddle angle on the incidence of low back pain in recreational bicyclists. British Journal of Sports Medicine, v. 33, n. I, p. 398-400, 1999.

SANNER, W. H.; O'HALLORAN, W. D. The biomechanics, etiology, and treatment of cycling injuries. Journal of the American Podiatric Medical Association, v. 90, n. 7, p. 354-376, 2000.

SCHULTZ S. J.; GORDON, S. J. Recreational cyclists - the relationship between low back pain and training characteristics. International Journal of Exercise Science, v. 3, n. 3 , p. $79-85,2010$.

SCHWELLNUS, M. P.; DERMAN, E. W. Common Injuries in Cycling: Prevention, Diagnosis and Management. South African Family Practice, v. 47, n. 7, p. 14-9, 2005.

SILBERMAN, M. R. et al. Road bicycle fit. Clinical Journal of Sport Medicine, v. I 5, n. 4, p. $27 \mid-276,2005$.

SRINIVASAN, J.; BALASUBRAMANIAN, V. Low back pain and muscle fatigue due to road cycling - an sEMG study. Journal of Bodywork and Movement Therapies, v. I I, n. 3, p. 260266, 2007.

SMURAWA, T. Overuse injuries curb triathlon preparation efforts. BioMechanics, v. 13, n. 5, p. 49-6I, 2006.

TROMBLEY, A. Serious mountain biking. I st ed. Champaign, IL: Human Kinetics, 2005.

USABIAGA. J. et al. Adaptation of the lumbar spine to different positions in bicycle racing. Spine (Phila Pa 1976), v. 22, n. 7, p. 1965-1969, 1997.

VILLAVICENCIO, A. T. et al. Back and neck pain in triathletes. Neurosurgical Focus, v. 2I, ก. 4, E7, 2006.

WILLARDSON, J. M. Core Stability Training for Healthy Athletes: A Different Paradigm for Fitness Professionals. National Strength and Conditioning Association, v. 29, n. 6, p. 42-49, 2007. 
WILBER, C. A. et al. An apidemiological analysis of overuse injuries among recreational cyclists international. Journal of Sports Medicine, v. 16, p. 201-206, 1995.

WILLY, R. Bicycling mechanics and proper fit. DRAYER Physical Therapy Institute, Fall, p. I-2, 2007.

XIANG, Z. et al. Optimal Design of Bicycle Frame Parameters Considering Biomechanics. Chinese Journal of Mechanical Engineering, v. 24, p. I-5, 201 I.

Recebido: 24 fev. 2010

Aprovado: 04 mar. 2011

Endereço para correspondência:

Thiago Ayala M. Di Alencar

Rua R-13, I59, Setor Oeste

Goiânia - GO - Brasil

CEP: 74.125-150 